\title{
Lithium treatment: prescribing and monitoring habits in hospital and general practice
}

\author{
R F Kehoe, A J Mander
}

\begin{abstract}
Objectives - To define current clinical practice of lithium prescribing and monitoring and to compare hospital based practice with general practice.

Design-Prospective study of doctors' practice.

Setting-Psychiatric hospital day and outpatient facilities and general practices in Edinburgh and Midlothian district (population 600000 ).

Subjects -458 patients taking lithium who had been stabilised and who remained as outpatients during the year of study. 219 were treated by their general practitioner and 190 by the hospital; 49 had shared care or care transferred during the study.

Main outcome measures-Daily dose, duration of treatment, psychiatric diagnosis, mean annual serum lithium concentration, frequency of occurrence of and response to raised serum concentrations.

Results-Compared with hospital doctors general practitioners were more likely to prescribe lithium three or more times daily (43/219 (general practice) $v$ $10 / 190$ (hospital); $\chi^{2}=18 \cdot 6, p=0 \cdot 001$ ) and to estimate serum concentrations less frequently (4.5 $v 5.3$ measurements/year; $t=3.04, p=0.003)$, and their patients were more likely to experience raised lithium concentrations $\left(39 / 219 v 17 / 190 ; \chi^{2}=6 \cdot 8, p=0.01\right)$. One third of doctors made no response to raised lithium concentrations in the next six weeks.

Conclusions-General practitioners and hospital doctors care for similar types of patients and the stringency of lithium surveillance varies greatly among doctors. Certain aspects of practice give cause for concern and could be improved by following more uniform guidelines.
\end{abstract}

\section{Introduction}

Lithium is widely used in the prophylaxis of manic depressive illness, and because of its pharmacokinetic and toxic properties regular monitoring of serum concentrations is recommended. Guidelines on how often serum concentrations should be measured vary from source to source: at the time of our study the British National Formulary suggested monthly estimations but now recommends estimations every three months.' Some authorities suggest six monthly estimations or less, 2 and most psychiatric texts recommend two monthly checks. ${ }^{3+}$ Medical insurance companies report that as many as $10 \%$ of claims for negligent psychiatric practice are associated with lithium treatment, particularly inadequate monitoring. ${ }^{5}$ When clinical guidelines vary acceptable practice is that performed by our peers. We conducted a study to define the standards of prescribing and monitoring by general

Royal Edinburgh Hospital, Edinburgh EH10 5HF R F Kehoe, MRCPSYCH, senior registrar

A J Mander, MRCPSYCH, lecturer

Correspondence to: $\mathrm{Dr}$ Kehoe.
Lothian Health Board is well suited for such a study as there is one NHS clinical chemistry laboratory in the area that performs serum lithium measurements and its result sheets form the basis of an established lithium register which contains details on all patients who have had serum lithium concentrations checked since $1970 .^{6}$ The laboratory interacts with practitioners in that reminders are sent to them regarding patients known to be taking lithium who have not had a serum estimation in the past 12 weeks. We prospectively collected a copy of the results of each serum lithium measurement (displaying details of practitioner, current dose, date and time of sample, and presence of side effects) over one year from June 1988. We established a new file which integrated information from the existing Edinburgh lithium register and the Lothian psychiatric case register. The case register records all patient contacts with psychiatric services in Edinburgh and Midlothian (population 600000), including basic demographic details, hospital admissions, and casenote psychiatric diagnosis based on the International Classification of Diseases (ninth revision). ${ }^{7}$ Further information on prescribed drug regimens and reasons for any dose changes implemented were acquired by postal communication with practitioners and occasionally with patients.

The local laboratory recommends a therapeutic range for lithium of $0 \cdot 6-1 \cdot 0 \mathrm{mmol} / \mathrm{l}$, and this is clearly stated on request and result sheets for lithium measurements. A concentration greater than $1.05 \mathrm{mmol} / \mathrm{l}$ was defined as above the therapeutic range. Mean annual serum lithium concentration was calculated in each case by dividing the sum of the concentrations by the number of estimations, excluding samples not taken 12-18 hours after the last dose. Duration of treatment was defined as continuous duration in years, without a break in lithium treatment of more than six months. The time in weeks between the data of a serum estimation that gave a result above the therapeutic range and the date of the next serum check was recorded. If the dose had been altered, the alteration was assumed to have occurred at the time of receipt of the abnormal result. Practitioners were contacted by post only after a dose had been altered to determine the reasons for that alteration. The laboratory contacts practitioners directly when serum concentrations are above $1.2 \mathrm{mmol} / \mathrm{l}$. Diagnosis was defined from the Lothian psychiatric case register as that at the time of last hospital discharge or last outpatient contact if the patient had had no admissions. For the purposes of data analysis diagnoses of circular manic depression subtypes were recoded as bipolar, and manic depressive psychosis depressed type, neurotic depression, and depressive disorder were recoded as unipolar affective disorder.

For inclusion in the study the patients had to meet the following criteria: firstly, they had to be resident in the area covered by Lothian psychiatric case register (Edinburgh lithium register covers a wider area); secondly, they had to have been taking lithium for longer than six months during the year of study (as frequency of serum estimation is inaccurate for patients taking lithium for only short periods); and, thirdly, they had to remain as outpatients during the year of study (as higher frequency of serum estimation during inpatient period would bias findings). Analysis was by the $\chi^{2}$ test for categorical variables and by Student's $t$ test and 
analysis of variance for continuous variables. Several variables were categorised for particular analyses namely, age $(<65 v \geqslant 65$ years) and annual serum lithium concentrations $(\geqslant 0.6 \mathrm{mmol} / \mathrm{l} v<0.6 \mathrm{mmol} / \mathrm{l})$.

\section{Results}

In all, 936 patients had serum lithium estimations during the year of study, giving a one year prevalence of $0 \cdot 16 \%$. A point prevalence of $0 \cdot 13 \%$ at the midpoint of the study year was estimated by excluding those who began lithium treatment after that time $(n=80)$ or who discontinued it in the first six months $(n=75)$. The inclusion criteria were met by 458 patients, of whom 219 were supervised by their general practitioner and 190 at psychiatric outpatient clinics; in the remaining 49 patients supervision was shared between the two or passed on during the year of study. The mean age of patients was $55 \cdot 8$ (SD 16) years (range 18-93 years) and $219(68 \%)$ were women. On average patients had had $3 \cdot 6$ psychiatric hospital admissions, with $9 \cdot 1$ years having elapsed since their first admission and 4.9 years since their last admission, and had been taking continuous lithium treatment for 6.8 years. Their mean annual serum lithium concentration was $0.69 \mathrm{mmol} / 1$, and they had an average of $5 \cdot 0(\mathrm{SD} 2 \cdot 8$; range $1-18)$ estimations a year. Two hundred and fifty two patients were taking lithium once daily, 146 twice daily, and 60 three or more times daily.

\section{SUPERVISON OF LITHIUM TREATMENT}

Comparisons of general practitioner and hospital based cases found no significant difference between these populations in age, sex, total number of admissions, time since first admission, diagnosis, and duration of treatment. Patients supervised by their general practitioner, however, were significantly more likely to receive lithium three or more times daily (43/ 219 (general practitioner) $v 10 / 190$ (hospital); $\chi^{i}=18 \cdot 6$, $\mathrm{df}=1, \mathrm{p}=0.001)$, had had more time since their last hospital admission $(5 \cdot 8 v 4 \cdot 4$ years; $t=2 \cdot 76, \mathrm{p}=0 \cdot 006)$, had a higher mean annual lithium concentration $(0 \cdot 71$ $v 0.66 \mathrm{mmol} / \mathrm{l} ; t=2.54, \mathrm{p}=0.01)$, and had their serum lithium concentration estimated less often $(4.5 v 5.3$ measurements/year; $t=3.04, \mathrm{p}=0.003$ ).

\section{VARIATION IN SERUM LITHIUM CONCENTRATIONS}

To determine the amount of variation within each patients' measurements, the proportion of patients whose lithium concentrations remained within the locally recommended therapeutic range and those who had concentrations above and below it were defined by using only those samples taken 12-18 hours after the last dose. In $181(39 \%)$ patients all the results of estimations of lithium concentration were within 0.6 $1.0 \mathrm{mmol} / \mathrm{l}$, in 55 patients at least one result was above this range, and in 196 at least one result was below. Twenty six $(6 \%)$ had results both above and below the range. Taking the wider range of $0.4-1.0 \mathrm{mmol} / \mathrm{l}$ (with a low point which is often clinically acceptable, especially for elderly patients), 309 patients had results within the range for all estimations, 73 had at least one result above the range, $68(15 \%)$ had one result below, and eight had results both above and below.

FREQUENCY OF RAISED LITHIUM CONCENTRATIONS AND DOCTORS' RESPONSE

There were three cases of serious lithium toxicity, two precipitated by physical illness (serum lithium 1.5 and $2.1 \mathrm{mmol} / \mathrm{l}$ ) and one (serum lithium $4.4 \mathrm{mmol} /$ 1) in a patient who had not had serum lithium concentrations checked for four years and who required emergency medical admission. Serum concentrations above the therapeutic range were detected in 56 patients, and these patients were more likely to be supervised by their general practitioner than at hospital clinics (39/219 (general practitioner) $v$ 17/190 (hospital); $\gamma^{\prime}=6.8, \mathrm{df}=1, \mathrm{p}=0.01$ ). Despite more patients in the general practitioner group having raised concentrations, general practitioners altered doses in significantly fewer patients than did hospital practitioners (38/219 (general practitioners) $v \quad 56 / 190$ (hospital); $\chi^{\prime}=8 \cdot 4, \mathrm{df}=1, \mathrm{p}=0 \cdot 01$ ). Most practitioners responded rapidly to high serum lithium concentrations but one third did not respond within the next six weeks. When doctors did respond the most common actions were to repeat the serum estimation or reduce the dosage, or both. The commonest reasons for changing the dose were high serum concentrations in the absence of clinical indicators, side effects, stabilising concentrations, aiming at concentrations nearer top or bottom of range, and high serum concentrations associated with toxic symptoms or predisposing conditions.

In 18 patients the lithium dose was reduced because of insidiously increasing lithium concentrations in the absence of any known predisposing circumstances or clinical signs. These patients were followed up in detail after the end of the study year as it is because of such cases that routine monitoring is recommended. Four of these patients were referred for specialist medical opinions because of lithium toxicity or renal complications either during the year of study or within the next six months.

\section{FREQUENCY OF SERUM LITHIUM ESTIMATIONS}

Patients in their first year of lithium treatment had serum estimations performed more frequently $(6.9 /$ year), but beyond this there was no significant change with increasing duration of treatment (average 5.0/year for treatment durations of 2-18 years). Elderly patients had their serum concentrations estimated more often than younger patients $(5 \cdot 4 /$ year $(\geqslant 65$ years) $v 4 \cdot 9 /$ year $(<65) ; t=4 \cdot 2 \mathrm{p}=0 \cdot 04)$. Patients who had their serum concentrations checked monthly or more often were compared with the rest of the population and did not differ significantly in any of the recorded measures.

Practitioners caring fur these patients were contacted by telephone to determine the reasons for such close monitoring. Most replied that they were following the guidelines in the British National Formulary (which at that time recommended monthly estimations), but several cited particular reasons such as poor compliance, concurrent diuretic treatment, poor renal function, or unstable psychiatric condition. Eighty five patients had serum concentrations checked only once or twice in the year, and these patients were more likely to be monitured by their general practitioner than in huspital (57/219 (general practitioner) $v 28 / 190$ (hospital); $\chi^{\prime}=7 \cdot 9, \mathrm{df}=1, \mathrm{p}=0.01$ ) and to have longer duration of treatment than the rest of the population ( 7.6 years (low frequency of serum checks) $v 6.7$ years (total population); $t=2 \cdot 7, \mathrm{p}=0.01$ ). The patients were similar in all other measures.

\section{PSYCHIAT RIC DIAGNOSIS}

Diagnosis was defined as bipolar affective disorder in 223 cases and unipolar disorder in 165 cases; other diagnoses included aggression, migraine, and other functional psychoses. Patients with bipolar disorders had had more hospital admissions (4.4 (bipolar) $v 2.9$ (unipular); $t=13.6, \mathrm{p}=0.001$ ) and more time had elapsed since their first admission $(10.2$ years $v 8.8$ ycars; $t=4.6, p=0.03$ ) when compared with patients with unipolar disorders. 'They also had longer durations of treatment ( $5 \cdot 1$ years (bipolar) $v 4 \cdot 4$ years (unipolar); $t=8 \cdot 1, \mathrm{p}=0.005)$. Neither the mean annual serum lithium concentration nor the frequency of serum estimations was related to diagnosis. 


\section{Discussion}

We identified all patients receiving lithium treatment within a defined region who had serum lithium concentrations measured during one year by using the results service of the local clinical chemistry department. Such a service enables identification of a particular clinical population and is a valuable source in the process of medical audit. Most studies of people taking lithium have been on patients attending specialised lithium clinics, which are attended only by selected patients. ${ }^{89}$ Two studies have attempted to identify all patients on lithium within defined areas and report a point prevalence of $0.08 \%$ in south west Scotland ${ }^{10}$ and $0 \cdot 15 \%$ in Sweden, ${ }^{11}$ similar to the $0 \cdot 13 \%$ in our study. There may be a few patients taking lithium who did not have a serum lithium estimation during the year of study, but the likelihood is reduced by the existence of the lithium register. Despite the presence of the register, and probably because of the differences in published guidelines, there is wide variation in the frequency with which different practitioners measure serum lithium concentrations. If peer practice is to be used as a standard, with hospital doctors checking serum concentrations every 10 weeks and general practitioners every 12 weeks, inadequate monitoring could be equated with checking less than six monthly, as observed in 22 cases in this region.

It should be noted that close surveillance of serum concentrations (monthly estimations) is recommended in certain subgroups of patients - for example those taking diuretics (thiazides, amiloride and the angiotensin converting enzyme inhibitors) and those with renal disease or poor renal function. ${ }^{12}$ In this survey, close monitoring mostly occurred because the doctor was following a particular set of guidelines rather than because of a specific indication.

The value of regular monitoring is illustrated by examination of cases in which serum concentrations rise in the absence of any known predisposing causes or clinical evidence of worsening side effects or toxicity. Unexplained high serum concentrations should be further assessed and investigated to detect causes such as declining renal function. (In an additional part of the study, which will be reported later, these patients were found to have significantly lower creatinine clearances than the rest of the group: $62.4 v 75.4 \mathrm{ml} / \mathrm{min}\left(1.73 \mathrm{~m}^{2}\right.$ surface area).) Given that individual variation is sufficient that 1 in 6 patients will experience a high result during one year, it is prudent to repeat the measurement before reducing the dose. A repeat measurement is best done within the next two weeks as the serum concentration may continue to rise. Although previous recommendations included wider therapeutic ranges, it is concerning that one third of doctors made no response to raised concentrations within six weeks. Regular monitoring, responding to high concentrations, and recognition of predisposing circumstances and early signs of toxicity by the patient and the practitioner can minimise the risk of serious toxicity.

The comparison of general practitioner and hospital based supervision reveals differences in the delivery of service, but this does not necessarily equate with inferiority of care. Patients had not been randomly allocated to their base of supervision, and given the similarity in demographic details and psychiatric history, it is difficult to determine the factors influencing this choice of care. This issue merits further study. Consistent standards of care are more easily delivered by specialised lithium clinics, which exist in many centres and have been well described, ${ }^{1314}$ and with the provision of equipment that enables instant measurement of serum lithium concentrations, delays in response to abnormal results could be minimised. ${ }^{\text {s }}$ Nevertheless, many patients and their doctors prefer lithium treatment to be supervised by their general practitioner or by individual psychiatrists, and in the current climate of health service development it is probable that increasing numbers of patients will be supervised by their general practitioner. This is the second study to find differences in the standards of lithium supervision between different parts of the health service, and hence it is important that all practitioners have a good understanding of current guidelines on the frequency of serum lithium estimations and reviews of thyroid, renal, and cardiac status.

\section{RECOMMENDATIONS}

Guidelines for dosage schedules and the frequency of serum lithium estimations need to be consistent and widely available. Locally this will be implemented by sending a form to practitioners along with annual review reminders from the lithium register. High serum lithium concentrations in the absence of predisposing conditions need to be investigated as they may be caused by declining renal function.

We thank the McGhie Fund; Mrs A Meikle; Mr R McGuire; Professor L Whitby; Drs A Smith, G Goodwin, D Blackwood, and L Whalley; and the medical records department at the Royal Edinburgh Hospital.

1 Joint Formulary Committee. British National Formulary No 22. London: British Medical Association/Pharmaceutical Society of Great Britain, 1991 Crammer J, Barraclough B, Heine B. The use of drugs in psychiatry. 2nd ed. London: Gaskell (Royal College of Psychiatrists), 1982.

3 Loudon JB. Drug treatment. In: Kendell RE, Zealley AK, eds. Companion to psychiatric studies. 4th ed. Edinburgh: Churchill Livingstone, 1988.

4 Gelder M, Gath D, Mayou R. Oxford textbook of psychiatry. 2nd ed. Oxford: Oxford University Press, 1989.

5 Medical Protection Society. Psychiatric negligence. Medical News 1989;1:2

6 Kehoe RF, Mander AJ. The Edinburgh lithium register. Psychiatric Bulletin of the Royal College of Psychiatrists 1989;13:611-2.

7 World Health Organisation. International classification of diseases, ninth revision. Geneva: WHO, 1978

8 Walker RG, Bennett WM, Davies BM, Kincaid-Smith P. Structural and functional effects of long term lithium therapy. Kidney Int 1982;21 (supp 11):513-9.

9 Gelenberg AJ, Kane JM, Keller MB, Lavori P, Rosenbaum JF, Cole K, et al. Comparison of standard and low serum levels of lithium for maintenance treatment of bipolar disorder. N Engl F Med 1989;321:1489-93.

10 McReadie RG, Morrison DP. The impact of lithium in south-west Scotland. 1. Demographic and clinical findings. Brf Psychiatry 1985;146:70-80.

11 Vestergaard $P$, Schou M. Lithium treatment in Aarhus. 1. Prevalence. Pharmacopsychiatry 1989;22:99-100.

12 DasGupta K, Jefferson JW. The use of lithium in the medically ill. Gen Hosp Psychiatry 1990;12:83-97.

13 Abou-Saleh $M$. Organising a lithium clinic. Practical Reziews in Psychiatr (Series 2) 1990;10:10-2.

It Gitlin MJ, Jamison KK. Lithium clinic: theory and practice. Hosp Community Psychiatry 1984;35:363-8.

15 King JR, Philips JD, Judge R, Armond AD, Corbett JA, Birch NJ. Instan lithium monitoring. Psychiatric Bulletin of the Roval College of Psychiatrist 1991;15:138-9.

(Accepted 3 December 1991) 